\title{
Estridor secundario a cuerpo extraño en esófago.
}

\section{Stridor secundary to an esophageal foreign body.}

Raquel Herrera-Rodríguez Gabriela Jiménez-Arguedas² José Pablo Gutiérrez Schwanhauser ${ }^{3}$

1,2 y 3 Médico Especialista. Caja costarricense seguro social, San José Costa Rica.

Contactos: gabyjimeneza@gmail.com

\section{RESUMEN}

Se trata de un masculino de 8 meses con historia de un mes de estridor que acude en múltiples ocasiones al servicio de emergencia sin mejoría. Se le realizaron dos radiografías de tórax que estaban normales. Por persistir sintomático se realizó broncoscopía que evidenció una compresión de la tráquea y posteriormente se visualiza un cuerpo extraño en esófago. Se lleva a sala de operaciones para gastroscopía y se logra extraer sin complicaciones una uña acrílica. No se documentaron perforaciones. El niño evoluciona satisfactoriamente. La ingesta inadvertida de un cuerpo extraño radiolúcido debe ser considerada dentro de las causas poco frecuentes de estridor.

Palabras Clave: Estridor; cuerpo extraño; lactante; esófago

\section{ABSTRACT}

The patient was an 8 month-old boy with history of 1 month of persistent stridor. He consulted multiple times to the emergency department with no improvement. He had to normal chest $x$-rays. Because of persistent symptoms he underwent a brocoscopy that visualized a tracheal compression and a foreign body in the esophagus. The

\section{Cómo citar:}

Herrera Rodríguez, R. ., Jiménez Arguedas, G., \& Gutiérrez Schwanhauser, J. P. . Estridor secundario a cuerpo extraño en esófago. Revista Ciencia Y Salud Integrando Conocimientos, 5(4), Pág. 87-91. https:// doi.org/10.34192/ cienciaysalud. v5i4.330

Recibido: 01/jun/2021 Aceptado: 01/sep/2021 Publicado: 04/sep/2021 pa-tient was transferred to de operating room for a gastroscopy and uneventful removal of an acrylic nail. He remained asymptomatic. Unvisualized ingestion of a radiolucent foreign body has to be considered in the differential diagnosis.

Keywords: Stridor; foreign body; infante; esophagus. 


\section{CIENCIA\&SALUD \\ ucime}

\section{CASO CLÍNICO}

Paciente masculino de 8 meses, conocido sano, sin antecedentes perinatales de im-portancia. Con historia de un mes de evolución de presentar estridor que había ido incrementando. Inicialmente era asociado con vómitos postprandiales, tos y rinorrea, pero posteriormente no presentaba ninguna otra sintomatología. Paciente consulta en repetidas ocasiones al servicio de emergencias donde es manejado con esteroides intramuscualres, orales, antibióticos y antitusivos, sin mejoría del cuadro. Además, contaba con dos radiografías de tórax sin hallazgos patológicos.

Ante la persistencia del estridor bifásico se realiza broncoscopía en la cual se descri-be a nivel de la tráquea una compresión extrínseca pulsátil en la región de la arteria innominada y además se observa cuerpo extraño en esófago; resto de estudio sin alteraciones (figura 1).

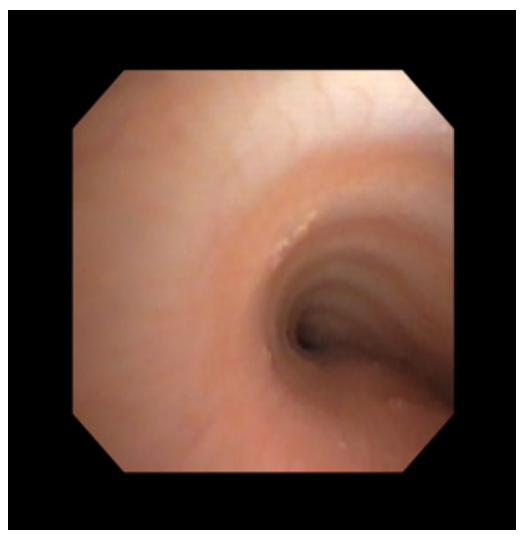

Figura 1. Broncoscopía donde se evidencia compresión extrínseca de la tráquea a nivel de arteria innominada.

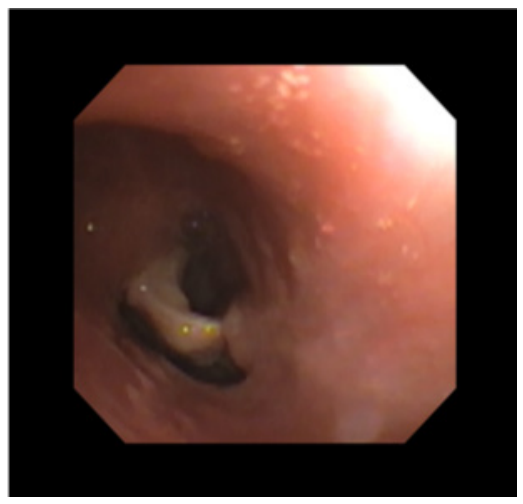

Figura 2. Gastroscopía con evidencia de cuerpo extraño a nivel de tercio superior de esófago.

El paciente es llevado a sala de operaciones para extracción de cuerpo extraño (CE), se realiza gastroscopía que evidencia a nivel de tercio superior del esófago una uña acrílica como la causante de una reacción de CE, lográndose extraer a primera inten-ción, sin complicaciones (figura 2 y 3). Posterior a procedimiento se realiza esofago-grama sin documentarse perforación esofágica.

Debido al hallazgo endoscópico de compresión extrínseca de la tráquea se realiza angiotac de tórax para descartar anillo vascular, sin embargo no se observa ninguna malformación vascular ni anatómica y se concluye que dicha compresión es secunda-ria a proceso inflamatorio por reacción de CE. 


\section{CIENCIA\&SALUD}

Un mes después de la extracción del CE, se realiza broncoscopía control con diag-nóstico de anatomía de la vía aérea normal. El paciente cursa totalmente asintomático.

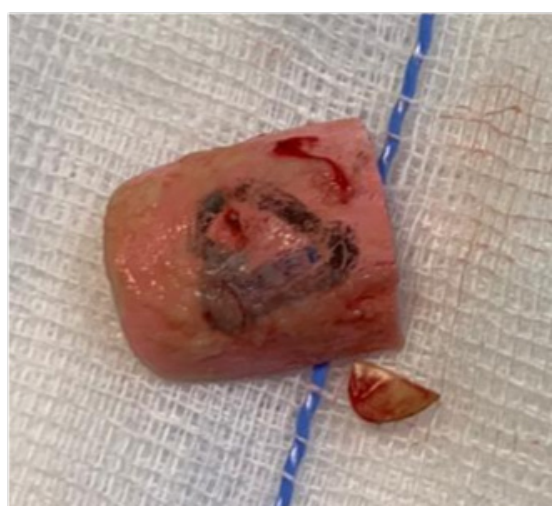

Figura 3. Uña acrílica extraída de esófago.

\section{Clasificación de espina bífida.}

La espina bífida oculta se debe a la falta de fusión de las mitades embrionarias de uno o más arcos neurales en la línea media, este defecto es común en las vértebras L5 o S1; este se puede observar como un pequeño penacho de pelos en la superficie indicando su presencia. La espina bífida quística contiene la protrusión de la médula espinal, meninges o ambas a través de los arcos vertebrales, formando una estructura llamada quiste meníngeo. Cuando el quiste contiene meninges y líquido cefalorraquídeo, el defecto se denomina espina bífida con meningocele (3).Si el tejido nervioso con las meninges están contenidos en el interior de un quiste meníngeo, el defecto se denomina espina bífida con mielomeningocele. El tipo más grave de espina bífida se denomina mielosquisis, el cual corresponde a falta de fusión de pliegues neurales, resultando en una médula espinal aplanada (3).

El nivel de lesión determina la zona dérmica sin inervación y los grupos musculares afectados. La parálisis de los esfínteres (vesical o anal) es frecuente en el mielomeningocele, acompañado a esto se produce una anestesia en silla de montar 4. Algunos casos de mielomeningocele se asocian a craniolacunia (desarrollo anómalo de la bóveda craneal); el resultado es la aparición de áreas de depresión no osificadas en las superficies internas de los huesos planos de la bóveda craneal (4). La mielosquisis suele cursar con parálisis o debilidad permanente de los miembros inferiores (4).

\section{DISCUSIÓN}

En 1962, se reportó el primer caso de ingestión de cuerpo extraño (CE) cuando un príncipe de 4 años, "Federico el Grande" se tragó la hebilla de su zapato (1). La ingesta de CE tiende a ocurrir usualmente en niños pequeños de 1 a 4 años (2,3). Esto se debe a su comportamiento exploratorio, control inmaduro de la de-glución y ausencia de molares y premolares (1). Los CE más comúnmente ingeri-dos o aspirados son las monedas y las baterías planas $(4,5)$.

La mayoría de los CE ingeridos atraviesan el tracto gastrointestinal sin complica-ciones (80\%), sin embargo, en ocasiones se alojan en lugares fisiológicos estre-chos. El esófago es uno de los sitios más comunes donde se localizan y se pre-sentan con sintomatología como sialorrea, disfagia o vómito. Cuando la ingesta pasa inadvertida y el CE en esófago se vuelve crónico, puede manifestarse hasta en un $72 \%$ con síntomas respiratorios como: tos, estridor, sibilancias, apnea y neumonía $(6,7)$. 
Pacientes con una ingestión no presenciada representan un desafío para el clí-nico. En este caso, el diagnóstico fue tardío debido a que el motivo de consulta fue estridor y no se sospechó de una ingesta de un CE. Las infecciones respira-torias y asma son causas frecuentes de estridor y sibilancias, mientras que un cuerpo extraño en esófago es raro. En la literatura se describen solamente 17 casos desde 1992 hasta 2012 (7). Un diagnóstico tardío en estos pacientes tiende a incrementar el riesgo de complicaciones (8). La retención de un cuerpo extraño en el esófago puede producir ulceración de la mucosa, inflamación y algunas complicaciones potencialmente mortales como absceso paraesofágico o retrofa-ríngeo, mediastinitis, empiema o perforaciones esofágicas(9).

Varios mecanismos han sido propuestos para explicar los síntomas respiratorios secundarios a un CE esofágico, debido a que la mayoría quedan retenidos a ni-vel del músculo cricofaríngeo (10). La proximidad de la vía aérea al esófago par-cialmente obstruido predispone a la aspiración traqueal y a la neumonía (11). También los síntomas respiratorios pueden ser secundarios a la compresión tra-queal por la presión directa de la pared posterior membranosa de la tráquea (que es particularmente blanda en lactantes y niños pequeños) por el propio CE o por la dilatación esofágica consecuente y a la extensión directa de la reacción inflamatoria a la laringe y la tráquea (11).

Si el CE se localiza por encima del músculo cricofaríngeo se recomienda larin-goscopía directa; para objetos localizados por debajo de este músculo el con-senso es utilizar endoscopio flexible (12). La gran mayoría se extraen sin compli-caciones, como este caso, pero dependiendo de la naturaleza y el tiempo de evolución se pueden presentar complicaciones como las anteriormente descritas.

Es fundamental que los profesionales de la salud alerten a los padres de los riesgos de la ingestión de un CE, los signos y síntomas posibles y la necesidad de atención médica inmediata. Los médicos no deben olvidar la posibilidad de un CE en un paciente con síntomas respiratorios que no responde al tratamiento usual.

\section{CONCLUSIONES}

La ingestión de CE en niños a menudo no se observa, puede ser asintomática y pue-de presentarse con síntomas y signos atípicos; por I o tanto, la sospecha clínica de ingestión de CE debe seguir siendo alta y ser considerada como diagnóstico diferen-cial de niños con estridor que no responden a tratamiento. Recordar que un diagnós-tico tardío se asocia con mayores complicaciones y morbilidades.

\section{REFERENCIAS BIBIOGRÁFICAS}

1. Denney W, Ahmad N, Dillard B, Nowicki M. Children will eat the strangest things: A 10-year retrospective analysis of foreign body and caustic ingestions from a single academic center. Pediatr Emer Care. 2012; 28(8): 731-734.

2. Cevik M, Gokdemir M, Boleken M, Sogut O, Kurkcuoglu C. The Characteristics and outcomes of foreign body ingestion and aspiration in children due to lodged foreign body in the aerodigestive tract. Pediatr Emer Care. 2013; 29(1): 53-57.

3. Kramer R, Lerner D, Lin T, Manfrendi $M$ et al. Management of Ingested Foreign Bodies in Children: A Clinical Report of the NASPGHAN Endoscopy Committee. J Pediatr Gastroenterol Nutr. 2015; 60(4): 562-574.

4. Kodituwakku R, Palmer S, Paul SP. Management of foreign body ingestions in children: button batteries and magnets. Br J Nurs. 2017;26(8):456-461. doi:10.12968/bjon.2017.26.8.456

5. Lešková J, Štichhauer R, Preis J, Šafus A, Koudelka J. Foreign body ingestion in children. Cizí tělesa v GIT u dětí. Rozhl Chir. 2019;98(9):370-374. doi:10.33699/PIS.2019.98.9.370-374. 


\section{CIENCIA\&SALUD}

6. Parray T, Shah S, Apuya JS, Shah S. Atypical presentation of an impacted radi-olucent esophageal foreign body. J Anesth 2010;24(5):793-6.

7. Hiejima E, Nakase H, Uemoto S, Heike T. Esophageal foreign body causing sustained stridor in an infant. Clin J Gastroenterol 2012;5(2):46-9.

8. Katsinelos P, Kountouras J, Paroutoglou G, Zavos C, Mimidis K, Chatzimavrou-dis G. Endoscopic Techniques and Management of Foreign Body Ingestion and Food Bolus Impaction in the Upper Gastrointestinal Tract: A Retrospective Analysis of 139 Cases. J Clin Gastroenterol. 2006; 40(9):784789

9. Hong K, Kim Y, Kim J, Chun S, Kim H, Cho J. Risk factors for complications as-sociated with upper gastrointestinal foreign bodies. World J Gastroenterol. 2015; 21(26): 8125-8131.

10. Rodríguez H, Passali GC, Gregori D, Chinski A, et al. Management of foreign bodies in the airway and oesophagus. Int J Pediatr Otorhinolaryngol 2012;76(Suppl 1):S84-91.

11. Garg A Wadhera R Gulati S et al. Esophageal foreign body presenting as repiratory distress. Internet J Pediatr Neonatol 2008;11(1):1-3.

12. Thomson M, Tringali A, Dumonceau JM, et al. Paediatric Gastrointestinal En-doscopy: European Society for Paediatric Gastroenterology Hepatology and Nutrition and European Society of Gastrointestinal Endoscopy Guidelines. J Pediatr Gastroenterol Nutr. 2017;64(1):133-153. doi:10.1097/ MPG.0000000000001408 